\title{
Dexametasona versus dimenhidrinato en la profilaxis de las náuseas y vómitos postoperatorios en pacientes adultos sometidos a cirugía general y digestiva
}

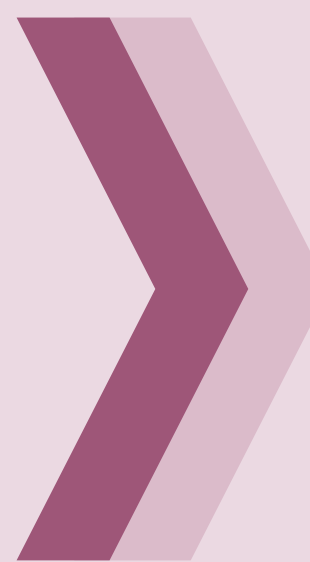

\author{
Dexamethasone versus \\ dimenhydrinate for prophylaxis of \\ postoperative nausea and vomiting \\ in adult patients undergoing \\ general and digestive surgery
}

\section{Carlos Shiraishi-Zapata ${ }^{1}$}

1. Médico asistente del servicio de Centro Quirúrgico y Anestesiología del Hospital II Essalud, Talara, Piura, Perú. Hospital Regional José Cayetano Heredia, Piura, Perú. Miembro de la Asociación de Anestesiología de la Región Piura (Asarep).

\section{RESUMEN}

INTRODUcción. Las náuseas y vómitos postoperatorios (NVPO) son complicaciones frecuentes en los pacientes quirúrgicos. Se han publicado una serie de guías clínicas y revisiones que recomiendan diferentes fármacos para la profilaxis y tratamiento de NVPO. Los fármacos dexametasona (DEX) y dimenhidrinato (DIM) son ampliamente utilizados para este fin; sin embargo, la evidencia científica que apoya la efectividad del DIM en el contexto de NVPO, es escasa. ОвJetivo. Determinar la dosis del fármaco (DIM o DEX con mayor efectividad en la profilaxis de náuseas y vómitos postoperatorios en pacientes adultos sometidos a cirugía general y laparoscópica. Asimismo, determinar la aparición de náuseas, vómitos, náuseas y vómitos postoperatorios, necesidad de tratamiento de rescate y efectos adversos de ambos medicamentos. Material y método. Ensayo clínico aleatorizado realizado en el Hospital II EsSalud de Talara, Piura, Perú con 102 participantes (18 hombres y 84 mujeres) con un riesgo bajo y moderado para NVPO (09 y 93 respectivamente), los que fueron asignados en dos grupos de 51 pacientes cada uno. Un grupo de pacientes recibió DEX (4 mg) y otro DIM (50 mg) luego de la inducción de la anestesia general. Resultados. La incidencia de NVPO en la población tratada con DEX fue de 7,84\% y de 39,22\% en la población de pacientes que recibieron DIM. Conclusiones. La administración de $4 \mathrm{mg}$ de dexametasona en el acto anestésico provee mejor profilaxis de náuseas y vómitos postoperatorios respecto a $50 \mathrm{mg}$ de dimenhidrinato.

Palabras clave: náuseas y vómitos postoperatorios; prevención y control; dexametasona; dimenhidrinato; cirugía general.

\begin{abstract}
BACKGround. Postoperative nausea and vomiting (PONV) are common complications in surgical patients. There have been a number of clinical guidelines and reviews that recommend different drugs for prophylaxis and treatment of PONV. Dexamethasone and dimenhydrinate drugs are widely used in our country for this purpose, but the scientific evidence supporting the effectiveness of dimenhydrinate in the context of PONV, is scarce. ОвנестIVE. To determine the dose of the drug (dimenhydrinate or dexamethasone) more effective in the prophylaxis of postoperative nausea and vomiting in adult patients undergoing general and laparoscopic surgery. Also determine the occurrence of nausea, vomiting, PONV, need for rescue treatment and adverse effects of both drugs. MateRIAL AND METHOD. Clinic randomized trial performed in the Hospital II Essalud Talara, Piura, Peru, with 102 participants (18 men and 84 women) with a low or moderate risk for $P O N V$ ( 09 and 93 respectively), which were assigned into two groups of 51 patients each. A group of patients received dexamethasone $(4 \mathrm{mg}$ ) and another dimenhydrinate $(50 \mathrm{mg})$ after induction of general anesthesia. RESULTS. The incidence of PONV in the dexamethasone-treated population was $7,84 \%$ and $39,22 \%$ in the population of patients who received dimenhydrinate. Conclusions. The dexamethasone administration of $4 \mathrm{mg}$ during anesthesia provides better prophylaxis of postoperative nausea and vomiting over dimenhydrinate administration of $50 \mathrm{mg}$.
\end{abstract}

KEYWORDS: postoperative nausea and vomiting; prevention and control; dexamethasone; dimenhydrinate; general surgery. 


\section{INTRODUCCIÓN}

Las náuseas y vómitos postoperatorios (NVPO), cuya etiología es multifactorial, son complicaciones frecuentes que producen malestar en el paciente, una estancia prolongada en la unidad de reanimación postanestésica (URPA) y, en pocas ocasiones, uno de los síndromes de aspiración pulmonar.

En la actualidad sigue siendo una de las principales complicaciones en el periodo postoperatorio con una incidencia media entre $20 \%$ y $30 \%$, pero que puede afectar hasta $80 \%$ de los pacientes. ${ }^{1}$

En los pacientes ambulatorios, las náuseas pueden afectar a $17 \%$ y los vómitos a $8 \%$. A pesar de que raramente es un problema grave, puede constituir un desagradable síntoma postoperatorio, que eventualmente retrasa el alta hospitalaria, disminuye la satisfacción del paciente y aumenta el consumo de recursos. Se estima que los vómitos adquieren características de gravedad en $0,1 \%$ a $0,2 \%$ de los casos por sus repercusiones hidroelectrolíticas, broncoaspiración, ruptura de suturas quirúrgicas, repercusiones neurológicas y agotamiento. ${ }^{2}$

Las Guías de la Sociedad de Anestesia Ambulatoria para el manejo de NVPO señalan que estos hechos son una preocupación continua en pacientes quirúrgicos y su manejo es aún confuso.

En EE UU, más de 71 millones de hospitalizaciones y procedimientos quirúrgicos ambulatorios se llevan a cabo cada año. Sin tratamiento, las náuseas y vómitos postoperatorios ocurren en $20 \%$ a $30 \%$ de la población quirúrgica general y en más de $70 \%$ a $80 \%$ de pacientes de alto riesgo. El rango de complicaciones de las NVPO va desde el agotamiento del paciente a la morbilidad postoperatoria. Las NVPO asociados a cirugía ambulatoria incrementan los costos de salud debido a la admisión hospitalaria y representa de $0,1 \%$ a $0,2 \%$ de estas admisiones no anticipadas, lo cual es significativo en EE UU, donde más de 31 millones de pacientes se someten a cirugía ambulatoria cada año. ${ }^{3}$

Se han publicado revisiones y guías respecto al manejo de náuseas y vómitos postoperatorias. ${ }^{3-12}$ En estas, se cita el uso de ambos fármacos, dexametasona y dimenhidrinato para la profilaxis de NVPO en pacientes adultos, con dosis de 4 a 10 $\mathrm{mg}$ en el caso de la dexametasona y de 1 a $2 \mathrm{mg}$ por kilogramo de peso para el dimenhidrinato.

Se ha llevado a cabo un metaanálisis de estudios controlados y aleatorizados para la profilaxis de náuseas y vómitos postoperatorios con dimenhidrinato por Kranke et al.,13 el cual concluye que este es un fármaco tradicional con una eficacia clínicamente relevante y que vale la pena considerar cuando se necesita un antiemético económico. Aunque ha estado en uso un largo tiempo, la dosis respuesta no está clara. Los efectos colaterales serios parecen ser raros.

Un segundo metaanálisis, denominado 'fármacos para la prevención de náuseas y vómitos postoperatorios', mucho más extenso en los estudios seleccionados y con una actualización continua, por Carlisle y Stevenson del Cochrane Anesthesia Group, ${ }^{14}$ pone en duda la efectividad de varios fármacos para este propósito, entre los que se encuentran dimenhidrinato, clonidina, hioscina, proclorperazina, ramosetrón, alizaprida, benzodiacepinas, perfenazina y prometazina. Cada antiemético se evaluó en su eficacia para prevenir de manera independiente: solo náuseas, solo vómitos, náuseas y vómitos de manera conjunta y eficacia del antiemético como fármaco de rescate en el período postoperatorio. Este meta-análisis incluyó 737 estudios con 103237 pacientes. En comparación con el placebo, los ocho fármacos siguientes previnieron las NVPO: droperidol, metoclopramida, ondansetrón, tropisetrón, dolasetrón, dexametasona, ciclizina y granisetrón. Concluye que todavía se encuentra por aclarar si son equivalentes entre ellos, y recomienda a los investigadores llevar a cabo estudios simples que comparen los 8 fármacos mencionados, ya que los resultados de tales estudios serán más fiables. ${ }^{14}$

En ese sentido, siendo que la dexametasona y dimenhidrinato son fármacos disponibles en los petitorios nacionales de medicamentos, económicamente accesibles y ampliamente conocidos en nuestro país, para la gran mayoría de pacientes que se someten a intervenciones quirúrgicas; de los que se tiene cierta experiencia para su uso en el ámbito del tratamiento y profilaxis de náuseas y vómitos, la presente investigación busca comparar la existencia de diferencias significativas en la incidencia de 
NVPO de los pacientes adultos sometidos a cirugía general y digestiva, provenientes de una población en la que se suministró dexametasona y otra en la que se suministró dimenhidrinato para la profilaxis de NVPO.

En el caso de dexametasona, se sabe que es un fármaco en el que la evidencia de manera unánime, confiere efectividad en la profilaxis, aunque no en el rescate, de NVPO. ${ }^{14}$

En el caso de dimenhidrinato, fue objeto de estudios en las décadas pasadas, ${ }^{7,11,16}$ e incluso de un metaanálisis, ${ }^{13}$ donde se concluía acerca de su utilidad en este contexto como se mencionó previamente. Sin embargo en el metaanálisis Cochrane de Carlisle ${ }^{14}$, este fármaco no logró una evidencia definitiva para su uso como profiláctico de NVPO. Este autor realizó una medición de la eficacia de los fármacos para profilaxis de NVPO a través de cuatro medidas. Cada antiemético se evaluó en su eficacia para prevenir de manera independiente: solo náuseas, solo vómitos, náuseas y vómitos de manera conjunta y eficacia del antiemético como fármaco de rescate en el postoperatorio. La intención de la presente investigación es comparar su eficacia con otro antiemético con establecida evidencia en la profilaxis de NVPO como es la dexametasona respecto a la incidencia de NVPO en tres primeras medidas, pues se utilizó otro antiemético de rescate (como tratamiento) en los pacientes en los que falló la profilaxis para NVPO de cualquiera de las dos poblaciones.

\section{MATERIAL Y MÉTODOS}

La presente investigación es un ensayo clínico aleatorizado, doble ciego, que analiza la incidencia de NVPO en dos poblaciones de pacientes de las que se obtuvieron dos muestras conformadas por 51 sujetos cada una; los mismos que fueron atendidos en el Hospital Essalud II Talara, Piura. La investigación es de tipo aplicada y también prospectiva, transversal, comparativa y observacional.

Se plantearon las siguientes hipótesis:

^ Hipótesisnula(Ho):laspoblaciones conformadas por pacientes a los que se les suministró de manera profiláctica dexametasona y dimenhidrinato, no presentan diferencias significativas respecto a la incidencia de NVPO.
- Hipótesis alterna (H1): las poblaciones conformadas por pacientes a los que se les suministró de manera profiláctica dexametasona y dimenhidrinato, presentan diferencias significativas respecto a la incidencia de NVPO.

Se incluyeron para el ensayo pacientes adultos (mayores de 19 años) de ambos sexos, sometidos a intervenciones quirúrgicas abdominales no ginecológicas programados para cirugía electiva y de emergencia, sometidos a anestesia general balanceada.

Asimismo con una puntuación de riesgo para NVPO correspondiente a riesgo bajo y moderado según la puntuación simplificada de Apfel para adultos. ${ }^{15}$

Se excluyeron de la investigación todos aquellos pacientes programados para intervenciones quirúrgicas extraabdominales (electivas y por emergencia). Asimismo, se excluyeron los pacientes sometidos a anestesia regional (epidural o raquídea), con una puntuación de riesgo alto para NVPO de acuerdo a la puntuación de Apfel, una historia de alergia a cualquiera de los fármacos, con tratamiento crónico con corticoides y/o dimenhidrinato por cualquier otra patología; diagnóstico $\mathrm{y} / 0$ tratamiento de enfermedad ulceropéptica de cualquier tipo; pacientes con un índice de masa corporal igual o mayor de treinta.

Los pacientes participantes de esta investigación (previa firma del consentimiento informado para la misma y seleccionados de acuerdo a los criterios de inclusión y exclusión) fueron aleatorizados (muestreo aleatorio sencillo) por el investigador en el área preoperatoria inmediata en dos grupos utilizando una tabla de números aleatorios, ${ }^{18}$ recibiendo el primer grupo $4 \mathrm{mg}$ de dexametasona inmediatamente después de la inducción anestésica; el grupo 2 recibió en el mismo intervalo $50 \mathrm{mg}$ de dimenhidrinato. La inducción se llevó a cabo con propofol, $2 \mathrm{mg} / \mathrm{kg}$; fentanilo, de 3 a $4 \mu \mathrm{g} /$ $\mathrm{kg}$, y rocuronio, $0,5 \mathrm{mg} / \mathrm{kg}$. El mantenimiento de la anestesia se llevó a cabo con sevoflurano y la analgesia se suministró mediante una combinación de un antiinflamatorio no esteroideo y un opioide (tramadol).

Se cegaron los fármacos en estudio para los pacientes, el anestesiólogo durante la cirugía y los investigadores que recolectaron los datos 
en el postoperatorio. Una enfermera de Centro quirúrgico tuvo la tarea de preparar los fármacos antieméticos en una jeringa quedando listos para su administración intravenosa. Se asignó dos colores distintos para cada tipo de fármaco antiemético, siendo elegidos el color verde y el color amarillo; de esta manera se solicitaba el antiemético de tal o cual color a ser administrado de acuerdo a la distribución aleatoria, sin que el investigador o colaboradores conocieran cuál de los dos antieméticos se suministraba. Al final de la investigación se reveló que medicamento tenía asignado el color verde 0 amarillo.

El seguimiento en el postoperatorio se llevó a cabo durante seis horas, pues las dosis aplicadas a ambos grupos fueron únicas y el tiempo de acción del dimenhidrinato se extiende hasta las seis horas aunque el de dexametasona sea prolongado. ${ }^{19}$ Se dividió el tiempo de seguimiento de manera arbitraria en tres períodos de observación, cada uno de los cuales fue de dos horas. El seguimiento se llevó a cabo en la URPA y en algunos casos en el área de hospitalización. Los pacientes de cualquiera de los dos grupos que presentaron cualquier evento adverso de NVPO recibieron Metoclopramida como tratamiento de rescate (20 mg). Se utilizó una ficha de recolección de datos para evaluar las variables de la investigación. Las entrevistas de los pacientes se llevaron a cabo por el investigador y los colaboradores de la investigación. Es importante señalar también que las arcadas se consideraron vómitos.

\section{Ética}

El protocolo de la presente investigación fue aprobado para su ejecución por el Comité de Ética del centro hospitalario. Asimismo, cada participante de la investigación firmó un consentimiento informado previamente al inicio de la misma. El protocolo completo también se encuentra en la Unidad de Posgrado de la Facultad de Medicina Humana de la Universidad Nacional Mayor de San Marcos, Lima, Perú.

\section{Estadística}

Para la selección de la muestra se tuvo en cuenta que para una población quirúrgica a la que se le suministra dimenhidrinato, se producirán NVPO en $61,2 \%$ de pacientes $^{16}$ y que la incidencia de
NVPO con dexametasona en pacientes de ambos sexos sometidos a cirugía en una investigación previa donde se suministró fue $23 \%{ }^{17}$ Se asumió un valor de $\alpha$ de 0,01 y uno de $\beta$ de 0,1 . Se calculó el respectivo valor $Z$ de ambos, considerando dos colas. La fórmula usada fue la siguiente:

$$
\mathrm{n}=(\mathrm{Z} \alpha+\mathrm{Z} \beta) 2(\mathrm{p} 1 \mathrm{q} 1+\mathrm{p} 2 \mathrm{q} 2) /(\mathrm{p} 1-\mathrm{p} 2)^{2}
$$

Al reemplazar:

$\mathrm{n}=(2,575+1,645) 2(61,2 \times 38,8+23 \times 77) /(61,2-23)^{2}$

De esta manera se obtiene un tamaño de muestra de 51 pacientes para cada grupo.

El análisis estadístico se llevó a cabo con el programa estadístico SPSS (SPSS Graduate Pack 15.0 for Windows). Se utilizó la prueba de ji cuadrado $\left(\chi^{2}\right)$ para evaluar la homogeneidad de las muestras provenientes de ambas poblaciones, encontrando si existen o no diferencias significativas entre estas respecto a la presencia de NVPO. Se utilizó un grado de libertad, y un valor de significación $\alpha$ de 0,01 ; con lo cual el valor crítico de $\chi^{2}$ es de 6,635.

El reclutamiento de los pacientes se llevó a cabo entre los meses de agosto de 2011 y junio de 2012. El seguimiento de los pacientes se efectuó durante el mismo período de tiempo como se detalla en la Figura 1.

\section{RESULTADOS}

La mayoría de pacientes que participaron de la investigación fueron de sexo femenino. En el grupo de 51 pacientes pertenecientes a la población a la que se suministró dexametasona, $13,73 \%$ (7 pacientes) fueron de sexo masculino y $86,27 \%$ (44), de sexo femenino. En el grupo de pacientes pertenecientes a la población tratada con dimenhidrinato, $21,57 \%$ (11 pacientes) fueron de sexo masculino y 78,43\% fueron de sexo femenino.

En lo que respecta al riesgo para presentar NVPO medido a través de la escala de riesgo según Apfel, $94,12 \%$ en la población a la que se suministró dexametasona tuvieron un riesgo moderado para presentar NVPO y $88,24 \%$ en la población que recibió dimenhidrinato. El porcentaje minoritario se clasificó como de riesgo bajo.

De acuerdo a la clasificación de estado físico según la American Society of Anesthesiologists (ASA), en la población tratada con dexametasona, 


\section{Anta Médica Pernana}

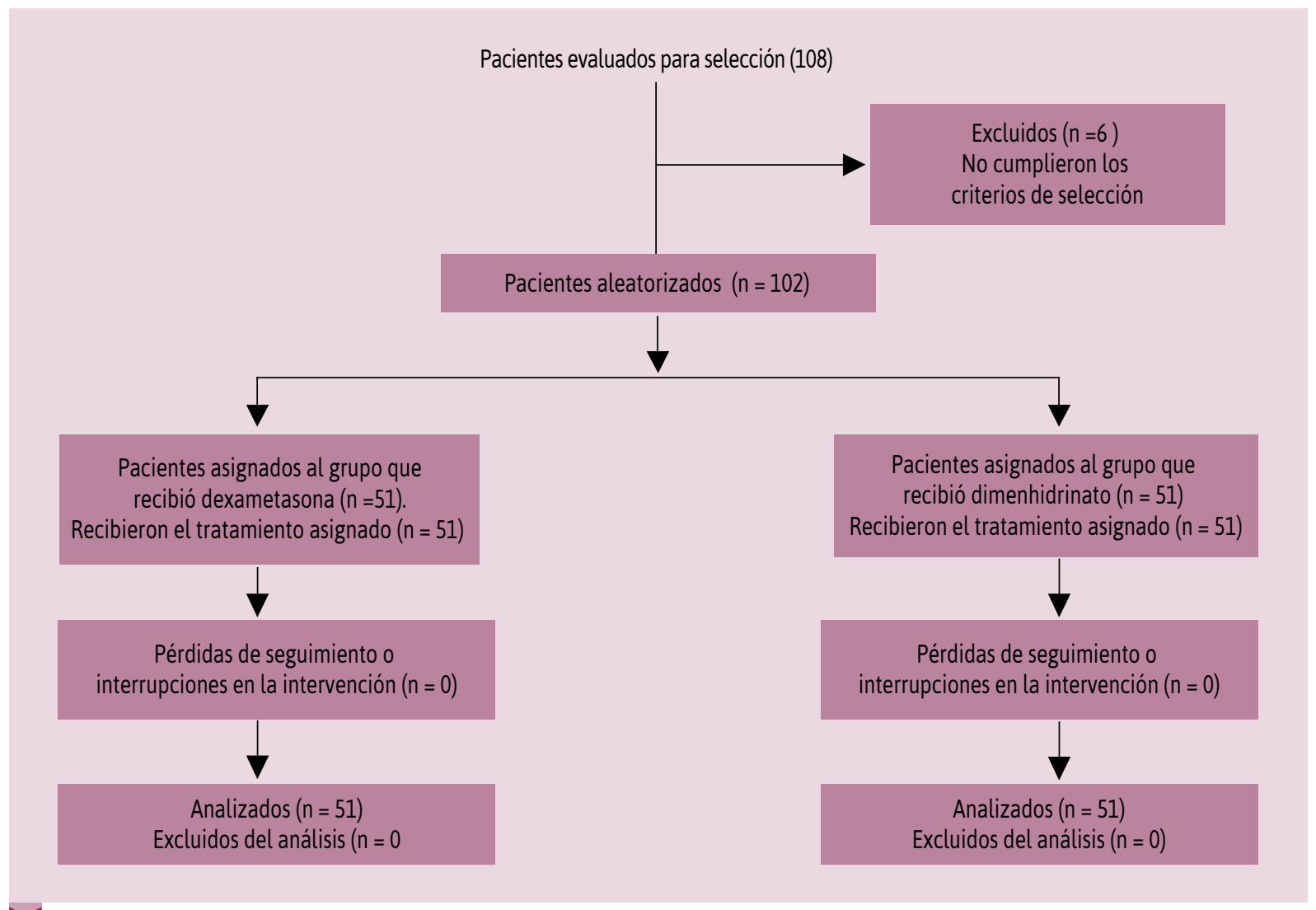

Figura 1. Seguimiento de los pacientes.

se obtuvo que 18,29 y 4 pacientes pertenecieron a las clases 1,2 y 3 , respectivamente. En la población asignada para recibir dimenhidrinato, se obtuvo que 29,18 y 4 pacientes fueron de las clases 1 , 2,3 , respectivamente. Asimismo de acuerdo a la asignación al índice de riesgo de Goldman, en la población que recibió dexametasona, 20 y 31 pacientes tuvieron una escala de riesgo de 1 y 2 respectivamente; en la otra población fueron 25 (clase 1) y 26 (clase 2 de Goldman).
El promedio de tiempo anestésico de los pacientes a los que se suministró dimenhidrinato fue de 106 minutos, y el tiempo quirúrgico fue de 82 minutos. En el caso de los tratados con dexametasona, fue de 104 y 80 minutos, respectivamente.

Los resultados de la incidencia de NVPO en la población tratada con dexametasona fueron de $7,84 \%$ y de $39,22 \%$ en los pacientes pertenecientes a la población que recibió como profilaxis dimenhidrinato (Tabla 1).

Tabla 1. Frecuencias observadas de náuseas y vómitos postoperatorios (NVPO) en los dos grupos de pacientes

\begin{tabular}{lccccc} 
Población & Presentaron NVPO & $\%$ & No presentaron NVPO & $\%$ & Total \\
$\Delta$ Dexametasona & 4 & 7,84 & 47 & 92,16 & 51 \\
Dimenhidrinato & 20 & 39,22 & 31 & 60,78 & 51 \\
Total & 24 & 23,53 & 78 & 76,47 & 102 \\
\hline
\end{tabular}


Tabla 2. Presencia de cualquier tipo de evento de náuseas y vómitos postoperatorios (NVPO) en el grupo tratado con dimenhidrinato 0 dexametasona, en las tres partes del período de observación

\begin{tabular}{|c|c|c|c|c|}
\hline \multirow{2}{*}{ Evento adverso } & \multicolumn{3}{|c|}{ Período } & \multirow[b]{2}{*}{ Total } \\
\hline & 0 a $2 \mathrm{~h}$ postoperatorias & 2 a $4 \mathrm{~h} \mathrm{postoperatorias}$ & $4 \mathrm{a} 6 \mathrm{~h}$ postoperatorias & \\
\hline \multicolumn{5}{|l|}{ D Dimenhidrinato } \\
\hline - Náuseas & 5 & 4 & 6 & 15 \\
\hline - Vómitos & 0 & 1 & 0 & 1 \\
\hline - Náuseas y vómitos & 4 & 3 & 6 & 13 \\
\hline - Vómitos sin náuseas & 1 & 0 & 0 & 1 \\
\hline Total & 10 & 8 & 12 & 30 \\
\hline \multicolumn{5}{|l|}{$\Delta$ Dexametasona } \\
\hline - Náuseas & 1 & 1 & 0 & 2 \\
\hline - Vómitos & 0 & 0 & 0 & 0 \\
\hline - Náuseas y vómitos & 1 & 1 & 0 & 2 \\
\hline - Vómitos sin náuseas & 0 & 0 & 0 & 0 \\
\hline Total & 2 & 2 & 0 & 4 \\
\hline
\end{tabular}

\section{Prueba de hipótesis}

De acuerdo a los resultados mostrados en la Tabla 1, se lleva a cabo la prueba de hipótesis utilizando una prueba de $\chi^{2}$ para homogeneidad de poblaciones, donde $\lambda$ es el valor del estadístico de Pearson. El contraste a utilizar sería el siguiente:

$\Delta$ Se acepta HO si $\lambda<\chi^{2}(\mathrm{r}-1)(\mathrm{s}-1) ; \alpha$

$\Delta$ Se rechaza Ho si $\lambda \geq \chi^{2}(r-1)(s-1) ; \alpha$

Se obtiene un valor $\lambda$ de 13,9487 que excede el valor de corte de $\chi^{2}$ con un grado de libertad que es de 6,635 (con la corrección de Yates se obtiene un valor de 12,2596 y un valor $p$ de 0,0004629 pero el valor utilizado para esta prueba de hipótesis se obtiene sin esta corrección, pues ninguna frecuencia esperada es mayor o igual a 5).

De esta manera se procede a rechazar la hipótesis de nulidad procediendo a aceptar que las poblaciones conformadas por pacientes a los que se les suministró de manera profiláctica dexametasona y dimenhidrinato, presentan diferencias significativas respecto a la incidencia de NVPO, o que no son homogéneas para la incidencia de NVPO. Asimismo, el valor $p$ que se obtiene es de 0,0001879 , lo que apoya el rechazo de la hipótesis de nulidad.

Respecto a los tres subperíodos del tiempo total de observación, en el caso de la población tratada con dimenhidrinato que es la que registra mayor incidencia de NVPO, se obtiene un leve predominio de los eventos de NVPO en el último de estos (12 eventos) como se aprecia en la Tabla 2. En la población que recibió dexametasona, los eventos de NVPO (4 en total) se presentaron en los dos primeros subperíodos, como se ve en la Tabla 2.

En lo que corresponde al tipo de NVPO, estuvo constituido de forma mayoritaria por las náuseas y también las náuseas y vómitos como se puede apreciar en la Tabla 3.

En la población de pacientes que recibieron dexametasona no se reportaron efectos adversos. En la población a la que se le suministró dimenhidrinato, siete pacientes los presentaron: tres de ellos manifestaron somnolencia, tres manifestaron mareos y uno de ellos, sequedad de boca.

Tabla 3. Número de pacientes que presentaron eventos de náuseas y vómitos postoperatorios (NVPO) en las poblaciones que recibieron profilaxis con ambos fármacos

$\begin{array}{lccc}\text { Tipo de evento } & \text { Dexametasona } & \text { Dimenhidrinato } & \text { Total } \\ \Delta \text { Náuseas } & 2 & 11 & 13 \\ \Delta \text { Vómitos } & 0 & 1 & 1 \\ \Delta \text { Náuseas y vómitos } & 2 & 8 & 10 \\ \Delta \text { Vómitos sin náuseas } & 0 & 1 & 1 \\ \text { Total } & 4 & 21^{*} & 25\end{array}$

* Un paciente presentó dos eventos distintos de NVPO. 


\section{DISCUSIÓN}

Las limitaciones de la presente investigación radican principalmente en el corto período de seguimiento ( 6 horas) a los pacientes participantes de la misma. El motivo de la elección de este período de observación fue la facilidad de observar a los pacientes en la URPA, evitando factores de confusión como el suministro de otros fármacos antieméticos y la valoración de las NVPO por otros miembros del personal de salud. Asimismo, la elección de pacientes considerados de bajo y moderado riesgo según la escala de Apfel obedece a la intención de hacer una valoración más clara de la acción de un único fármaco contra otro, ya que los pacientes considerados de alto riesgo, de acuerdo a las guías y revisiones publicadas, requerirían un abordaje multimodal y el uso de dos fármacos para la profilaxis de NVPO; si se les suministrara solamente uno, podríamos estar cometiendo una falta contra la ética al exponer pacientes a un evento adverso y dar un tratamiento profiláctico incompleto con un solo fármaco antiemético. 3,4,11

En la presente investigación, se ha obtenido una incidencia de NVPO en el grupo tratado con dexametasona menor a la citada en la literatura. En el caso de la población de pacientes tratados con dimenhidrinato, esta supera levemente a los datos encontrados en la literatura ( $20 \%$ a $30 \%)$, aunque sin tratamiento el porcentaje de pacientes afectados por NVPO llegaría hasta $80 \%{ }^{2}$

Se procedió a informar con detalle el tipo de evento y los distintos eventos de NVPO en los tres subperíodos de observación, según las implicaciones para la investigación consignadas en el metaanálisis de fármacos para la prevención de náuseas y vómitos postoperatorios de Carlisle y Stevenson. ${ }^{14}$

No se aprecia una concordancia entre el número de eventos de NVPO y el número de pacientes que tuvieron profilaxis con dimenhidrinato, porque en algunos casos los pacientes tuvieron episodios de NVPO en dos o más períodos de observación; por lo que el número de eventos de NVPO reportados, supera al número de pacientes (Tabla 2). De manera que se reportan 30 eventos de NVPO en distintos períodos de observación, pero esa cantidad de eventos se produjo en 21 pacientes (pero un paciente presentó dos eventos distintos, con lo que el número se reduce a 20); en el grupo tratado con dexametasona, se presentaron cuatro eventos, que se presentaron en cuatro pacientes.

Los pacientes que presentaron cualquier tipo de NVPO recibieron tratamiento de rescate con otro fármaco antiemético (metoclopramida).

La prueba estadística de $\chi^{2}$ explicada en la sección de resultados, establece claramente la aceptación de que existe una diferencia significativa, una ausencia de homogeneidad en la incidencia de efectos adversos tipo NVPO entre las dos poblaciones de pacientes, mostrando claramente un menor porcentaje de pacientes afectados en la población a la que se suministró dexametasona.

De acuerdo con esto, y teniendo en cuenta las conclusiones del metaanálisis sobre fármacos para la prevención de NVPO realizado por Carlisle, en el que no hallaba evidencia de la efectividad del dimenhidrinato para una profilaxis efectiva; los resultados de la presente investigación concuerdan con la conclusión sobre la duda acerca de la efectividad de ciertos medicamentos antieméticos, entre los que se halla el dimenhidrinato, para tratar náuseas y vómitos en el contexto postoperatorio. Una pregunta que surge luego de la presente investigación es la siguiente: ¿el uso de una dosis mayor de dimenhidrinato ofrecería una profilaxis efectiva de NVPO a diferencia del precario efecto en la profilaxis con una dosis del fármaco de $50 \mathrm{mg}$ ?

Los efectos adversos no fueron de gravedad e incluso estuvieron ausentes en la población que recibió dexametasona.

\section{Agradecimientos}

A la investigadora participante, Ysabel Cubas Castillo. Interna de Medicina, Hospital II Essalud Talara, Piura, por su participación en el la obtención y recogimiento de datos.

A los colaboradores: Dra. Yovanky More Vilela, Dra. Karina Córdova Martínez, Dr.Augusto Vásquez, Dr. Telésforo León Velasco, Dr. Robert Rivas Anastasio, por su participación en la evaluación de los pacientes en los períodos de observación; Lic. Rebeca Luna Córdova, Lic. Deysa Herreda Abad, por su participación en la preparación de los medicamentos profilácticos a ser suministrados; Dra. Melva Urbina Guillén y Dr. Edhi Alcalde Sisniegas, por la aprobación del protocolo para ser ejecutado en el correspondiente servicio hospitalario. 


\section{REFERENCIAS BIBLIOGRÁFICAS}

I. Feeley T, Macario A. Unidad de Reanimación post-anestésica. En: Fleisher L, Johns R, Savarese J, Wiener-Kronish J, Young W. Miller Anestesia. Sexta edición. Madrid: Elsevier; 2005. p. 2703-2728.

2. Álvarez García J, Castillo Monsegur J, Escolano Villén F. Motilidad gastrointestinal: fisiología del vómito y la regurgitación. En: Fisiología aplicada a la Anestesiología. Tomo I. Segunda edición. Fundación Europea para la enseñanza de la Anestesiología en la formación continuada; 2005. p. 293-316.

3. Gan TJ, Meyer TA, Apfel CC, Chung F, Davis PJ, Habib AS, et al. Society for Ambulatory Anesthesia Guidelines for Management of Postoperative Nausea and Vomiting.Anesth Analg. 2007;105;6I5-28.

4. McCraken G, Houston P, Lefebvre G. Guidelines for the management of Postoperative Nausea and Vomiting.J Obstet Gynaecol Can. 2008; 30(7):600-607.

5. Gan TJ. Management of Postoperative Nausea and Vomiting. 2009. Chapter 6. Volume 37. Rosenblatt M, Butterworth J, Gross J, editors. ASA Refresher Course in Anesthesiology.

6. Salamanca Montaña M, López Álvarez S, Cobian Llamas JM. Náuseas y vómitos Post-operatorios en cirugía ambulatoria. Cir May Amb. 2005;10(I): 13-18.

7. Bucley DN, Tramèr MR. Prevention: Dimenhydrinate prevents postoperative nausea and vomiting. Can JAnesth. 2003;50(I):II-I3.

8. Tramèr MR. A rational approach to the control of postoperative nausea and vomiting: evidence from systematic reviews. Part I. Efficacy and harm of antiemetic interventions, and methodological issues. Acta Anaesthesiol Scand. 200I;45(I): 4-13.

9. Tramèr MR. A rational approach to the control of postoperative nausea and vomiting: evidence from systematic reviews. Part II. Recommendations for prevention and treatment, and research agenda.Acta Anaesthesiol Scand. 200 I;45(I):14-19.

10. Habib A, Gan T. Evidence-based management of postoperative nausea and vomiting: a review. Can JAnesth. 2004;5I (4):326-34I.
1I. Hume RH, Wilner WK Jr. The use of dramamine in control of postoperative nausea and vomiting.Anesthesiology. 1952; 13(3):302-5.

12. Bel Marcoval I, Gambús Cerrillo P. Estratificación del riesgo, profilaxis y tratamiento de las náuseas y vómitos postoperatorios. Rev Esp Anestesiol Reanim. 2006; 53 (5): 30I-3I I.

13. Kranke P, Morin M, Roewer N, Eberhart H. Dimenhydrinate for prophylaxis of postoperative nausea and vomiting: a meta-analysis of randomized controlled trials.Acta Anaesthesiol Scand. 2002;46(3): 238-244.

14. Carlisle JB, Stevenson CA. Fármacos para la prevención de náuseas y vómitos postoperatorios (Cochrane Review). In: La Biblioteca Cochrane Plus [Revista on-line] 2008 (3) [acceso 09 de Abril de 2009]. Oxford: Update Software. DOI: 10.1002//465/858. CD004I25.pub2. Disponible en: http://onlinelibrary.wiley.com/ doi/10.1002/14651858.CD004/25.pub2/pdf/standard

15. Apfel CC, Laara E, Koivuranta M, Greim CA, Roewer N.A simplified risk score for predicting postoperative nausea and vomiting. Anesthesiology. 1999;91(3):693-700.

16. Eberhart LH, Seeling W, Bopp TI, Morin AM, Georgieff M. Dimenhydrinate for prevention of post-operative nausea and vomiting in female in-patients. Eur J Anaesthesiol. 1999; 16(5):284-9.

17. Nesek-Adam V, Grizelj-Stojcić E, Rasić Z, Cala Z, Mrsić V, Smiljanić A. Comparison of dexamethasone, metoclopramide and their combination in the prevención of postoperative nausea and vomiting after laparoscopic cholecistectomy. Surg Endosc. 2007;2I (4): 607-12.

18. Dawson B,Trapp R. Bioestadística Médica.Tercera edición. México: El Manual Moderno; 2002 p. I0I-I44, 367-369.

19. Cobos-Carbó A, Augustovski F. Declaración CONSORT 2010: actualización de la lista de comprobación para informar ensayos clínicos aleatorizados de grupos paralelos. CONSORT 2010 Declaration: Updated guideline for reporting parallel group randomized trials. Med Clin (Barc). 20I I;137(5):2।3-2I5.

\section{Correspondencia \\ Dr. Carlos Shiraishi-Zapata \\ shiraishi52@hotmail.com}

Fecha de recepción: 18 de setiembre de 2014

Fecha de aceptación: 25 de setiembre de 2014

\section{Conflicto de intereses}

El autor declara no tener ningún conflicto de intereses. 